Mathematical Sciences And Applications E-Notes

Volume 2 No. 1 PP. 55-67 (2014) @ MSAEN

\title{
ON GENERALIZATION OF DIFFERENT TYPE INTEGRAL INEQUALITIES FOR $s$-CONVEX FUNCTIONS VIA FRACTIONAL INTEGRALS
}

\author{
IMDAT İSCAN
}

(Communicated by Nihal Yılmaz ÖZGÜR)

\begin{abstract}
In this paper, a new general identity for differentiable mappings via Riemann-Liouville fractional integrals has been defined. By using of this identity, author has obtained new estimates on generalization of Hadamard, Ostrowski and Simpson type inequalities for functions whose derivatives in absolutely value at certain powers are $s$-convex in the second sense.
\end{abstract}

\section{INTRODUCTION}

Following inequalities are well known in the literature as Hermite-Hadamard inequality, Ostrowski inequality and Simpson inequality respectively:

Theorem 1.1. Let $f: I \subseteq \mathbb{R} \rightarrow \mathbb{R}$ be a convex function defined on the interval $I$ of real numbers and $a, b \in I$ with $a<b$. The following double inequality holds

$$
f\left(\frac{a+b}{2}\right) \leq \frac{1}{b-a} \int_{a}^{b} f(x) d x \leq \frac{f(a)+f(b)}{2} .
$$

Theorem 1.2. Let $f: I \subseteq \mathbb{R} \rightarrow \mathbb{R}$ be a mapping differentiable in $I^{\circ}$, the interior of $I$, and let $a, b \in I^{\circ}$ with $a<b$. If $\left|f^{\prime}(x)\right| \leq M, x \in[a, b]$, then we the following inequality holds

$$
\left|f(x)-\frac{1}{b-a} \int_{a}^{b} f(t) d t\right| \leq \frac{M}{b-a}\left[\frac{(x-a)^{2}+(b-x)^{2}}{2}\right]
$$

for all $x \in[a, b]$.

Theorem 1.3. Let $f:[a, b] \rightarrow \mathbb{R}$ be a four times continuously differentiable mapping on $(a, b)$ and $\left\|f^{(4)}\right\|_{\infty}=\sup _{x \in(a, b)}\left|f^{(4)}(x)\right|<\infty$. Then the following inequality

Date: Received: September 19, 2013; Accepted: April 11, 2014.

2010 Mathematics Subject Classification. 26A51, 26A33, 26D10.

Key words and phrases. Hermite-Hadamard inequality, Riemann-Liouville fractional integral, Ostrowski inequality, Simpson type inequality, $s$-convex function. 
holds:

$$
\left|\frac{1}{3}\left[\frac{f(a)+f(b)}{2}+2 f\left(\frac{a+b}{2}\right)\right]-\frac{1}{b-a} \int_{a}^{b} f(x) d x\right| \leq \frac{1}{2880}\left\|f^{(4)}\right\|_{\infty}(b-a)^{4} .
$$

In [8], Hudzik and Maligranda considered among others the class of functions which are $s$-convex in the second sense.

Definition 1.1. A function $f:[0, \infty) \rightarrow \mathbb{R}$ is said to be $s$-convex in the second sense if

$$
f(\alpha x+\beta y) \leq \alpha^{s} f(x)+\beta^{s} f(y)
$$

for all $x, y \in[0, \infty), \alpha, \beta \geq 0$ with $\alpha+\beta=1$ and for some fixed $s \in(0,1]$. This class of $s$-convex functions in the second sense is usually denoted by $K_{s}^{2}$.

It can be easily seen that for $s=1, s$-convexity reduces to ordinary convexity of functions defined on $[0, \infty)$.

In [6], Dragomir and Fitzpatrick proved a variant of Hermite-Hadamard inequality which holds for the $s$-convex functions.

Theorem 1.4. Suppose that $f:[0, \infty) \rightarrow[0, \infty)$ isans-convex function in the second sense, where $s \in(0,1]$ and let $a, b \in[0, \infty), a<b$. If $f \in L[a, b]$, then the following inequalities hold

$$
2^{s-1} f\left(\frac{a+b}{2}\right) \leq \frac{1}{b-a} \int_{a}^{b} f(x) d x \leq \frac{f(a)+f(b)}{s+1}
$$

the constant $k=\frac{1}{s+1}$ is the best possible in the second inequality in (1.2). The above inequalities are sharp.

In [9], Iscan obtained inequalities for differentiable convex mapping which are connected Simpson's inequality, and he used the following lemma to prove this

Lemma 1.1. Let $f: I \subseteq \mathbb{R} \rightarrow \mathbb{R}$ be a differentiable mapping on $I^{\circ}$ such that $f^{\prime} \in L[a, b]$, where $a, b \in I$ with $a<b$ and $\theta, \lambda \in[0,1]$. Then the following equality holds:

$$
\begin{aligned}
& (1-\theta)(\lambda f(a)+(1-\lambda) f(b))+\theta f((1-\lambda) a+\lambda b)-\frac{1}{b-a} \int_{a}^{b} f(x) d x \\
= & (b-a)\left[-\lambda^{2} \int_{0}^{1}(t-\theta) f^{\prime}(t a+(1-t)[(1-\lambda) a+\lambda b]) d t\right. \\
& \left.+(1-\lambda)^{2} \int_{0}^{1}(t-\theta) f^{\prime}(t b+(1-t)[(1-\lambda) a+\lambda b]) d t\right] .
\end{aligned}
$$

The main inequalities in [9], pointed out, are as follows.

Theorem 1.5. Let $f: I \subseteq[0, \infty) \rightarrow \mathbb{R}$ be a differentiable mapping on $I^{\circ}$ such that $f^{\prime} \in L[a, b]$, where $a, b \in I^{\circ}$ with $a<b$ and $\theta, \lambda \in[0,1]$. If $\left|f^{\prime}\right|^{q}$ is s-convex on 
$[a, b], q \geq 1$, then the following inequality holds:

$$
\begin{gathered}
\left|(1-\theta)(\lambda f(a)+(1-\lambda) f(b))+\theta f((1-\lambda) a+\lambda b)-\frac{1}{b-a} \int_{a}^{b} f(x) d x\right| \\
\leq \quad(b-a) A_{1}^{1-\frac{1}{q}}(\theta)\left\{\lambda^{2}\left[\left|f^{\prime}(a)\right|^{q} A_{2}(\theta, s)+\left|f^{\prime}(C)\right|^{q} A_{3}(\theta, s)\right]^{\frac{1}{q}}\right. \\
\left.+(1-\lambda)^{2}\left[\left|f^{\prime}(b)\right|^{q} A_{2}(\theta, s)+\left|f^{\prime}(C)\right|^{q} A_{3}(\theta, s)\right]^{\frac{1}{q}}\right\}
\end{gathered}
$$

where

$$
\begin{aligned}
A_{1}(\theta) & =\theta^{2}-\theta+\frac{1}{2} \\
A_{2}(\theta, s) & =\frac{2 \theta^{s+2}}{(s+1)(s+2)}-\frac{\theta}{s+1}+\frac{1}{s+2} \\
A_{3}(\theta, s) & =\frac{2(1-\theta)^{s+2}}{(s+1)(s+2)}-\frac{1-\theta}{s+1}+\frac{1}{s+2} .
\end{aligned}
$$

and $C=(1-\lambda) a+\lambda b$.

Theorem 1.6. Let $f: I \subseteq[0, \infty) \rightarrow \mathbb{R}$ be a differentiable mapping on $I^{\circ}$ such that $f^{\prime} \in L[a, b]$, where $a, b \in I^{\circ}$ with $a<b$ and $\theta, \lambda \in[0,1]$. If $\left|f^{\prime}\right|^{q}$ is s-convex on $[a, b], q>1$, then the following inequality holds:

$$
\begin{aligned}
& \left|(1-\theta)(\lambda f(a)+(1-\lambda) f(b))+\theta f((1-\lambda) a+\lambda b)-\frac{1}{b-a} \int_{a}^{b} f(x) d x\right| \\
& \leq \quad(b-a)\left(\frac{\theta^{p+1}+(1-\theta)^{p+1}}{p+1}\right)^{\frac{1}{p}} \\
& \quad \times\left[\lambda^{2}\left(\frac{\left|f^{\prime}(a)\right|^{q}+\left|f^{\prime}(C)\right|^{q}}{s+1}\right)^{\frac{1}{q}}+(1-\lambda)^{2}\left(\frac{\left|f^{\prime}(b)\right|^{q}+\left|f^{\prime}(C)\right|^{q}}{s+1}\right)^{\frac{1}{q}}\right] .
\end{aligned}
$$

where $C=(1-\lambda) a+\lambda b$ and $\frac{1}{p}+\frac{1}{q}=1$.

We give some necessary definitions and mathematical preliminaries of fractional calculus theory which are used throughout this paper.

Definition 1.2. Let $f \in L[a, b]$. The Riemann-Liouville integrals $J_{a^{+}}^{\alpha} f$ and $J_{b^{-}}^{\alpha} f$ of oder $\alpha>0$ with $a \geq 0$ are defined by

$$
J_{a^{+}}^{\alpha} f(x)=\frac{1}{\Gamma(\alpha)} \int_{a}^{x}(x-t)^{\alpha-1} f(t) d t, x>a
$$

and

$$
J_{b^{-}}^{\alpha} f(x)=\frac{1}{\Gamma(\alpha)} \int_{x}^{b}(t-x)^{\alpha-1} f(t) d t, x<b
$$


respectively, where $\Gamma(\alpha)$ is the Gamma function defined by $\Gamma(\alpha)=\int_{0}^{\infty} e^{-t} t^{\alpha-1} d t$ and $J_{a^{+}}^{0} f(x)=J_{b^{-}}^{0} f(x)=f(x)$.

In the case of $\alpha=1$, the fractional integral reduces to the classical integral. Properties concerning this operator can be found $[7,11,14]$.

In recent years, many athors have studied error estimations for Hermite-Hadamard, Ostrowski and Simpson inequalities on the class of $s$-convex functions in the second sense; for refinements, counterparts, generalization see $[2,3,5,9,10,12,13,16,17$, $18,19,20,21]$.

The main aim of this article is to establish new generalization of HermiteHadamard type, Ostrowski type and Simpson-type inequalities for functions whose derivatives in absolutely value at certain powers are $s$-convex in the second sense. These results have some relationships with [9] for $\alpha=1$. To begin with the author will derive a general integral identity for differentiable mappings via fractional integral.

\section{Generalized integral InEQUALities for $s$-CONVEX FUnCtions Via} FRACTIONAL INTEGRALS

Let $f: I \subseteq \mathbb{R} \rightarrow \mathbb{R}$ be a differentiable function on $I^{\circ}$, the interior of $I$, throughout this section we will take

$$
\begin{aligned}
S_{f}(x, \mu, \alpha ; a, b)= & (1-\mu)\left[\frac{(x-a)^{\alpha}+(b-x)^{\alpha}}{b-a}\right] f(x)+\mu\left[\frac{(x-a)^{\alpha} f(a)+(b-x)^{\alpha} f(b)}{b-a}\right] \\
& -\frac{\Gamma(\alpha+1)}{b-a}\left[J_{x^{-}}^{\alpha} f(a)+J_{x^{+}}^{\alpha} f(b)\right]
\end{aligned}
$$

where $a, b \in I$ with $a<b, \quad x \in[a, b], \mu \in[0,1], \alpha>0$ and $\Gamma$ is Euler Gamma function. In order to prove our main results we need the following identity.

Lemma 2.1. Let $f: I \subseteq \mathbb{R} \rightarrow \mathbb{R}$ be a differentiable function on $I^{\circ}$ such that $f^{\prime} \in L[a, b]$, where $a, b \in I$ with $a<b$. Then for all $x \in[a, b], \mu \in[0,1]$ and $\alpha>0$ we have:

$$
\begin{aligned}
& S_{f}(x, \mu, \alpha ; a, b)=\frac{(x-a)^{\alpha+1}}{b-a} \int_{0}^{1}\left(t^{\alpha}-\mu\right) f^{\prime}(t x+(1-t) a) d t \\
& +\frac{(b-x)^{\alpha+1}}{b-a} \int_{0}^{1}\left(\mu-t^{\alpha}\right) f^{\prime}(t x+(1-t) b) d t .
\end{aligned}
$$


Proof. By integration by parts and changing the variable, for $x \neq a$ we can state

$$
\begin{aligned}
& \int_{0}^{1}\left(t^{\alpha}-\lambda\right) f^{\prime}(t x+(1-t) a) d t \\
= & \left.\left(t^{\alpha}-\mu\right) \frac{f(t x+(1-t) a)}{x-a}\right|_{0} ^{1}-\int_{0}^{1} \alpha t^{\alpha-1} \frac{f(t x+(1-t) a)}{x-a} d t \\
= & (1-\mu) \frac{f(x)}{x-a}+\mu \frac{f(a)}{x-a}-\frac{\alpha}{x-a} \int_{a}^{x}\left(\frac{u-a}{x-a}\right)^{\alpha-1} \frac{f(u)}{x-a} d u \\
= & (1-\mu) \frac{f(x)}{x-a}+\mu \frac{f(a)}{x-a}-\frac{\Gamma(\alpha+1)}{(x-a)^{\alpha+1}} J_{x^{-}}^{\alpha} f(a)
\end{aligned}
$$

and for $x \neq b$ similarly we get

$$
\begin{aligned}
& \int_{0}^{1}\left(\mu-t^{\alpha}\right) f^{\prime}(t x+(1-t) b) d t \\
= & \left.\left(\mu-t^{\alpha}\right) \frac{f(t x+(1-t) b)}{x-b}\right|_{0} ^{1}-\int_{0}^{1} \alpha t^{\alpha-1} \frac{f(t x+(1-t) b)}{x-b} d t \\
= & (1-\mu) \frac{f(x)}{b-x}+\mu \frac{f(b)}{b-x}-\frac{\alpha}{b-x} \int_{x}^{b}\left(\frac{b-u}{b-x}\right)^{\alpha-1} \frac{f(u)}{b-x} d u \\
= & (1-\mu) \frac{f(x)}{b-x}+\mu \frac{f(b)}{b-x}-\frac{\Gamma(\alpha+1)}{(b-x)^{\alpha+1}} J_{x^{+}}^{\alpha-f(b)}
\end{aligned}
$$

Multiplying both sides of $(2.2)$ and $(2.3)$ by $\frac{(x-a)^{\alpha+1}}{b-a}$ and $\frac{(b-x)^{\alpha+1}}{b-a}$, respectively, and adding the resulting identities we obtain the desired result.

For $x=a$ and $x=b$ the identities

$$
S_{f}(a, \mu, \alpha ; a, b)=(b-a)^{\alpha} \int_{0}^{1}\left(\mu-t^{\alpha}\right) f^{\prime}(t a+(1-t) b) d t
$$

and

$$
S_{f}(b, \mu, \alpha ; a, b)=(b-a)^{\alpha} \int_{0}^{1}\left(t^{\alpha}-\mu\right) f^{\prime}(t b+(1-t) a) d t
$$

can be proved easily by performing an integration by parts in the integrals from the right side and changing the variable.

Theorem 2.1. Let $f: I \subseteq[0, \infty) \rightarrow \mathbb{R}$ be a differentiable function on $I^{\circ}$ such that $f^{\prime} \in L[a, b]$, where $a, b \in I^{\circ}$ with $a<b$. If $\left|f^{\prime}\right|^{q}$ is s-convex on $[a, b]$ for some fixed $q \geq 1, x \in[a, b], \mu \in[0,1]$ and $\alpha>0$ then the following inequality for fractional 
integrals holds

$$
\begin{aligned}
& \left|S_{f}(x, \mu, \alpha ; a, b)\right| \\
(2.4) \leq & A_{1}^{1-\frac{1}{q}}(\alpha, \mu)\left\{\frac{(x-a)^{\alpha+1}}{b-a}\left(\left|f^{\prime}(x)\right|^{q} A_{2}(\alpha, \mu, s)+\left|f^{\prime}(a)\right|^{q} A_{3}(\alpha, \mu, s)\right)^{\frac{1}{q}}\right. \\
+ & \left.\frac{(b-x)^{\alpha+1}}{b-a}\left(\left|f^{\prime}(x)\right|^{q} A_{2}(\alpha, \mu, s)+\left|f^{\prime}(b)\right|^{q} A_{3}(\alpha, \mu, s)\right)^{\frac{1}{q}}\right\},
\end{aligned}
$$

where

$$
\begin{aligned}
A_{1}(\alpha, \mu) & =\frac{2 \alpha \mu^{1+\frac{1}{\alpha}}+1}{\alpha+1}-\mu, \\
A_{2}(\alpha, \mu, s) & =\frac{2 \alpha \mu^{1+\frac{s+1}{\alpha}}+s+1}{(s+1)(\alpha+s+1)}-\frac{\mu}{s+1}, \\
A_{3}(\alpha, \mu, s) & =\mu\left[\frac{1-2\left(1-\mu^{\frac{1}{\alpha}}\right)^{s+1}}{s+1}\right]+\beta(\alpha+1, s+1)-2 \beta\left(\mu^{\frac{1}{\alpha}} ; \alpha+1, s+1\right),
\end{aligned}
$$

$\beta$ is Euler Beta function defined by

$$
\beta(x, y)=\frac{\Gamma(x) \Gamma(y)}{\Gamma(x+y)}=\int_{0}^{1} t^{x-1}(1-t)^{y-1} d t, \quad x, y>0
$$

and

$$
\beta(a, x, y)=\int_{0}^{a} t^{x-1}(1-t)^{y-1} d t, \quad 0<a<1, x, y>0,
$$

is incomplete Beta function.

Proof. From Lemma 2.1, property of the modulus and using the power-mean inequality we have

$$
\begin{aligned}
& \left|S_{f}(x, \mu, \alpha, a, b)\right| \leq \frac{(x-a)^{\alpha+1}}{b-a} \int_{0}^{1}\left|t^{\alpha}-\mu\right|\left|f^{\prime}(t x+(1-t) a)\right| d t \\
& +\frac{(b-x)^{\alpha+1}}{b-a} \int_{0}^{1}\left|\mu-t^{\alpha}\right|\left|f^{\prime}(t x+(1-t) b)\right| d t \\
& \leq \quad \frac{(x-a)^{\alpha+1}}{b-a}\left(\int_{0}^{1}\left|t^{\alpha}-\mu\right| d t\right)^{1-\frac{1}{q}}\left(\int_{0}^{1}\left|t^{\alpha}-\mu\right|\left|f^{\prime}(t x+(1-t) a)\right|^{q} d t\right)^{\frac{1}{q}} \\
& \quad+\frac{(b-x)^{\alpha+1}}{b-a}\left(\int_{0}^{1}\left|t^{\alpha}-\mu\right| d t\right)^{\frac{1}{q}}\left(\int_{0}^{1}\left|t^{\alpha}-\mu\right|\left|f^{\prime}(t x+(1-t) b)\right|^{q} d t\right)^{\frac{1}{q}}
\end{aligned}
$$


Since $\left|f^{\prime}\right|^{q}$ is $s$-convex in the second sense on $[a, b]$ we get

$$
\begin{aligned}
\int_{0}^{1}\left|t^{\alpha}-\mu\right|\left|f^{\prime}(t x+(1-t) a)\right|^{q} d t & \leq \int_{0}^{1}\left|t^{\alpha}-\mu\right|\left(t^{s}\left|f^{\prime}(x)\right|^{q}+(1-t)^{s}\left|f^{\prime}(a)\right|^{q}\right) d t \\
& =\left|f^{\prime}(x)\right|^{q} A_{2}(\alpha, \mu, s)+\left|f^{\prime}(a)\right|^{q} A_{3}(\alpha, \mu, s), \\
\int_{0}^{1}\left|t^{\alpha}-\mu\right|\left|f^{\prime}(t x+(1-t) b)\right|^{q} d t & \leq \int_{0}^{1}\left|t^{\alpha}-\mu\right|\left(t^{s}\left|f^{\prime}(x)\right|^{q}+(1-t)^{s}\left|f^{\prime}(b)\right|^{q}\right) d t \\
(2.7) & =\left|f^{\prime}(x)\right|^{q} A_{2}(\alpha, \mu, s)+\left|f^{\prime}(b)\right|^{q} A_{3}(\alpha, \mu, s),
\end{aligned}
$$

where we use the fact that

$$
\begin{aligned}
\int_{0}^{1}\left|t^{\alpha}-\mu\right|(1-t)^{s} d t= & \int_{0}^{\mu^{\frac{1}{\alpha}}}\left(\mu-t^{\alpha}\right)(1-t)^{s} d t+\int_{\mu^{\frac{1}{\alpha}}}^{1}\left(t^{\alpha}-\mu\right)(1-t)^{s} d t \\
= & \mu \int_{0}^{\mu^{\frac{1}{\alpha}}}(1-t)^{s} d t-\int_{0}^{\mu^{\frac{1}{\alpha}}} t^{\alpha}(1-t)^{s} d t+\int_{\mu^{\frac{1}{\alpha}}}^{1} t^{\alpha}(1-t)^{s} d t \\
& \left.-\mu \int_{\mu^{\frac{1}{\alpha}}(1-t)^{s} d t}^{1}\right]+\int_{0}^{1} t^{\alpha}(1-t)^{s} d t-2 \int_{0}^{\mu^{\frac{1}{\alpha}}} t^{\alpha}(1-t)^{s} d t \\
= & \mu\left[\frac{1-2\left(1-\mu^{\frac{1}{\alpha}}\right)^{s+1}}{s+1}\right]+\beta(\alpha+1, s+1)-2 \beta\left(\mu^{\frac{1}{\alpha}} ; \alpha+1, s+1\right), \\
= & \mu\left[\frac{1-2\left(1-\mu^{\frac{1}{\alpha}}\right)^{s+1}}{s+1}\right]+\frac{2 \alpha \mu^{1+\frac{s+1}{\alpha}}+s+1}{(s+1)(\alpha+s+1)}-\frac{\mu}{s+1}, \\
\int_{0}^{1}\left|t^{\alpha}-\mu\right| t^{s} d t= &
\end{aligned}
$$

and by simple computation

$$
\begin{aligned}
\int_{0}^{1}\left|t^{\alpha}-\mu\right| d t & =\int_{0}^{\mu^{\frac{1}{\alpha}}}\left(\mu-t^{\alpha}\right) d t+\int_{\mu^{\frac{1}{\alpha}}}^{1}\left(t^{\alpha}-\mu\right) d t \\
& =\frac{2 \alpha \mu^{1+\frac{1}{\alpha}}+1}{\alpha+1}-\mu .
\end{aligned}
$$

Hence, If we use (2.6), (2.7) and (2.8) in (2.5), we obtain the desired result. This completes the proof.

Remark 2.1. In Theorem 2.1, if we take $\alpha=1, \mu=1-\theta$ and $x=(1-\lambda) a+\lambda b$ with $\lambda \in[0,1]$, then we obtain the same of the inequality (1.3) in Theorem 1.5. 
Corollary 2.1. In Theorem 2.1, if we take $s=1$, then the inequality (2.4) reduces to the following inequality

$$
\begin{aligned}
& \left|S_{f}(x, \mu, \alpha, a, b)\right| \\
\leq & A_{1}^{1-\frac{1}{q}}(\alpha, \mu)\left\{\frac{(x-a)^{\alpha+1}}{b-a}\left(\left|f^{\prime}(x)\right|^{q} A_{2}(\alpha, \mu, 1)+\left|f^{\prime}(a)\right|^{q} A_{3}(\alpha, \mu, 1)\right)^{\frac{1}{q}}\right. \\
& \left.+\frac{(b-x)^{\alpha+1}}{b-a}\left(\left|f^{\prime}(x)\right|^{q} A_{2}(\alpha, \mu, 1)+\left|f^{\prime}(b)\right|^{q} A_{3}(\alpha, \mu, 1)\right)^{\frac{1}{q}}\right\} .
\end{aligned}
$$

Corollary 2.2. In Theorem 2.1, if we take $q=1$, then the inequality (2.4) reduces to the following inequality

$$
\begin{aligned}
\left|S_{f}(x, \mu, \alpha, a, b)\right| \leq & \left\{\frac{(x-a)^{\alpha+1}}{b-a}\left(\left|f^{\prime}(x)\right| A_{2}(\alpha, \mu, s)+\left|f^{\prime}(a)\right| A_{3}(\alpha, \mu, s)\right)\right. \\
& \left.+\frac{(b-x)^{\alpha+1}}{b-a}\left(\left|f^{\prime}(x)\right| A_{2}(\alpha, \mu, s)+\left|f^{\prime}(b)\right| A_{3}(\alpha, \mu, s)\right)\right\} .
\end{aligned}
$$

Corollary 2.3. In Theorem 2.1, if we take $x=\frac{a+b}{2}$ and $\mu=\frac{1}{3}$, then the inequality (2.4) reduces the following Simpson type inequality for fractional integrals

$$
\begin{aligned}
& \left|\frac{2^{\alpha-1}}{(b-a)^{\alpha-1}} S_{f}\left(\frac{a+b}{2}, \frac{1}{3}, \alpha, a, b\right)\right| \\
= & \mid \frac{1}{6}\left[f(a)+4 f\left(\frac{a+b}{2}\right)+f(b)\right]-\frac{\Gamma(\alpha+1) 2^{\alpha-1}}{(b-a)^{\alpha}}\left[J_{\left(\frac{a+b}{2}\right)^{-}}^{\alpha}(a)+J_{\left.\left(\frac{a+b}{2}\right)^{+} f(b)\right] \mid}^{\alpha} \leq\right. \\
\leq & \frac{b-a}{4} A_{1}^{1-\frac{1}{q}}\left(\alpha, \frac{1}{3}\right)\left\{\left(\left|f^{\prime}\left(\frac{a+b}{2}\right)\right|^{q} A_{2}\left(\alpha, \frac{1}{3}, s\right)+\left|f^{\prime}(a)\right|^{q} A_{3}\left(\alpha, \frac{1}{3}, s\right)\right)^{\frac{1}{q}}\right. \\
& \left.+\left(\left|f^{\prime}\left(\frac{a+b}{2}\right)\right|^{q} A_{2}\left(\alpha, \frac{1}{3}, s\right)+\left|f^{\prime}(b)\right|^{q} A_{3}\left(\alpha, \frac{1}{3}, s\right)\right)^{\frac{1}{q}}\right\} .
\end{aligned}
$$

Corollary 2.4. In Theorem 2.1, if we take $x=\frac{a+b}{2}$ and $\mu=0$ then the inequality (2.4) reduces the following midpoint inequality for fractional integrals

$$
\begin{aligned}
& \left|\frac{2^{\alpha-1}}{(b-a)^{\alpha-1}} S_{f}\left(\frac{a+b}{2}, 0, \alpha, a, b\right)\right| \\
= & \left|f\left(\frac{a+b}{2}\right)-\frac{\Gamma(\alpha+1) 2^{\alpha-1}}{(b-a)^{\alpha}}\left[J_{\left(\frac{a+b}{2}\right)^{-}}^{\alpha} f(a)+J_{\left(\frac{a+b}{2}\right)}^{\alpha} f(b)\right]\right| \\
\leq & \frac{b-a}{4}\left(\frac{1}{\alpha+1}\right)^{1-\frac{1}{q}}\left\{\left[\frac{\left|f^{\prime}\left(\frac{a+b}{2}\right)\right|^{q}}{\alpha+s+1}+\left|f^{\prime}(a)\right|^{q} \beta(\alpha+1, s+1)\right]^{\frac{1}{q}}\right. \\
& \left.+\left[\frac{\left|f^{\prime}\left(\frac{a+b}{2}\right)\right|^{q}}{\alpha+s+1}+\left|f^{\prime}(b)\right|^{q} \beta(\alpha+1, s+1)\right]^{\frac{1}{q}}\right\} .
\end{aligned}
$$

Remark 2.2. In Corollary 2.4, if we take $\alpha=1$ and use the inequality (1.2), then we obtain the same of the inequality in [3, Theorem 2.4]. 
Corollary 2.5. In Theorem 2.1, if we take $\mu=1$ then the inequality (2.4) reduces the following trapezoid inequality for fractional integrals

$$
\begin{aligned}
& \left|S_{f}\left(\frac{a+b}{2}, 1, \alpha, a, b\right)\right| \\
= & \left|\frac{(x-a)^{\alpha} f(a)+(b-x)^{\alpha} f(b)}{b-a}-\frac{\Gamma(\alpha+1)}{b-a}\left[J_{x^{-}}^{\alpha} f(a)+J_{x^{+}}^{\alpha} f(b)\right]\right| \\
\leq & \left(\frac{\alpha}{\alpha+1}\right)^{1-\frac{1}{q}}\left\{\frac{(x-a)^{\alpha+1}}{b-a}\left[\frac{\alpha\left|f^{\prime}(x)\right|^{q}}{(s+1)(\alpha+s+1)}+\left|f^{\prime}(a)\right|^{q}\left(\frac{1}{s+1}-\beta(\alpha+1, s+1)\right)\right]^{\frac{1}{q}}\right. \\
& \left.+\frac{(b-x)^{\alpha+1}}{b-a}\left[\frac{\alpha\left|f^{\prime}(x)\right|^{q}}{(s+1)(\alpha+s+1)}+\left|f^{\prime}(b)\right|^{q}\left(\frac{1}{s+1}-\beta(\alpha+1, s+1)\right)\right]^{\frac{1}{q}}\right\}
\end{aligned}
$$

which is the same of the inequality in [12, Theorem 9].

Remark 2.3. In Corollary 2.5, if we choose $\alpha=1$, we get the same inequality in [4, Theorem 7].

Corollary 2.6. Let the assumptions of Theorem 2.1 hold. If $\left|f^{\prime}(x)\right| \leq M$ for all $x \in[a, b]$ and $\mu=0$, then from the inequality (2.4) we get the following Ostrowski type inequality for fractional integrals

$$
\begin{aligned}
& \left|\left[\frac{(x-a)^{\alpha}+(b-x)^{\alpha}}{b-a}\right] f(x)-\frac{\Gamma(\alpha+1)}{b-a}\left[J_{x^{-}}^{\alpha} f(a)+J_{x^{+}}^{\alpha} f(b)\right]\right| \\
\leq & M\left(\frac{1}{\alpha+1}\right)^{1-\frac{1}{q}}\left[\frac{1}{\alpha+s+1}+\beta(\alpha+1, s+1)\right]^{\frac{1}{q}}\left[\frac{(x-a)^{\alpha+1}+(b-x)^{\alpha+1}}{b-a}\right]
\end{aligned}
$$

for each $x \in[a, b]$.

Remark 2.4. In Corollary 2.6, if we choose $\alpha=1$, we get the same inequality in [2, Theorem 4].

Theorem 2.2. Let $f: I \subseteq[0, \infty) \rightarrow \mathbb{R}$ be a differentiable function on $I^{\circ}$ such that $f^{\prime} \in L[a, b]$, where $a, b \in I^{\circ}$ with $a<b$. If $\left|f^{\prime}\right|^{q}$ is s-convex on $[a, b]$ for some fixed $q>1$, then for $x \in[a, b], \mu \in[0,1]$ and $\alpha>0$ the following inequality for fractional integrals holds

$$
\begin{aligned}
& \left|S_{f}(x, \mu, \alpha ; a, b)\right| \\
\leq & A_{4}^{\frac{1}{p}}(\alpha, \mu, p)\left\{\frac{(x-a)^{\alpha+1}}{b-a}\left(\frac{\left|f^{\prime}(x)\right|^{q}+\left|f^{\prime}(a)\right|^{q}}{s+1}\right)^{\frac{1}{q}}\right. \\
+ & \left.\frac{(b-x)^{\alpha+1}}{b-a}\left(\frac{\left|f^{\prime}(x)\right|^{q}+\left|f^{\prime}(b)\right|^{q}}{s+1}\right)^{\frac{1}{q}}\right\},
\end{aligned}
$$

where

$$
\begin{aligned}
& A_{4}(\alpha, \mu, p)
\end{aligned}
$$

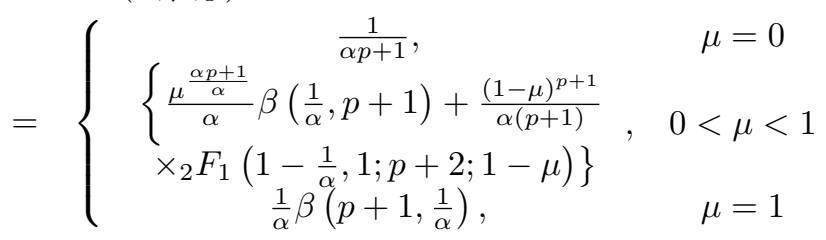


and ${ }_{2} F_{1}$ is hypergeometric function defined by ${ }_{2} F_{1}(a, b ; c ; z)=\frac{1}{\beta(b, c-b)} \int_{0}^{1} t^{b-1}(1-t)^{c-b-1}(1-z t)^{-a} d t, c>b>0,|z|<1$ (see [1]).

Proof. From Lemma 2.1, property of the modulus and using Hölder inequality we have

$$
\begin{aligned}
& \left|S_{f}(x, \mu, \alpha, a, b)\right| \leq \frac{(x-a)^{\alpha+1}}{b-a} \int_{0}^{1}\left|t^{\alpha}-\mu\right|\left|f^{\prime}(t x+(1-t) a)\right| d t \\
& +\frac{(b-x)^{\alpha+1}}{b-a} \int_{0}^{1}\left|\mu-t^{\alpha}\right|\left|f^{\prime}(t x+(1-t) b)\right| d t \\
& \leq \frac{(x-a)^{\alpha+1}}{b-a}\left(\int_{0}^{1}\left|t^{\alpha}-\mu\right|^{p} d t\right)^{\frac{1}{p}}\left(\int_{0}^{1}\left|f^{\prime}(t x+(1-t) a)\right|^{q} d t\right)^{\frac{1}{q}} \\
& +\frac{(b-x)^{\alpha+1}}{b-a}\left(\int_{0}^{1}\left|t^{\alpha}-\mu\right|^{p} d t\right)^{\frac{1}{p}}\left(\int_{0}^{1}\left|f^{\prime}(t x+(1-t) b)\right|^{q} d t\right)^{\frac{1}{q}}
\end{aligned}
$$

Since $\left|f^{\prime}\right|^{q}$ is $s$-convex in the second sense on $[a, b]$ we get

$$
\begin{aligned}
\int_{0}^{1}\left|f^{\prime}(t x+(1-t) a)\right|^{q} d t & \leq \int_{0}^{1} t^{s}\left|f^{\prime}(x)\right|^{q}+(1-t)^{s}\left|f^{\prime}(a)\right|^{q} d t \\
& =\frac{\left|f^{\prime}(x)\right|^{q}+\left|f^{\prime}(a)\right|^{q}}{s+1},
\end{aligned}
$$

$$
\begin{aligned}
\int_{0}^{1}\left|f^{\prime}(t x+(1-t) b)\right|^{q} d t & \leq \int_{0}^{1} t^{s}\left|f^{\prime}(x)\right|^{q}+(1-t)^{s}\left|f^{\prime}(b)\right|^{q} d t \\
& =\frac{\left|f^{\prime}(x)\right|^{q}+\left|f^{\prime}(b)\right|^{q}}{s+1},
\end{aligned}
$$

and by simple computation

$$
\begin{aligned}
\int_{0}^{1}\left|t^{\alpha}-\mu\right|^{p} d t & \mu=0 \\
= & \left\{\begin{array}{cc}
\frac{1}{\alpha p+1}, & \\
\left\{\begin{array}{cc}
\frac{\mu^{\frac{\alpha p+1}{\alpha}}}{\alpha} \beta\left(\frac{1}{\alpha}, p+1\right)+\frac{(1-\mu)^{p+1}}{\alpha(p+1)} \\
\left.\times{ }_{2} F_{1}\left(1-\frac{1}{\alpha}, 1 ; p+2 ; 1-\mu\right)\right\} \\
\frac{1}{\alpha} \beta\left(p+1, \frac{1}{\alpha}\right),
\end{array}\right. & \mu<\mu<1
\end{array}\right.
\end{aligned}
$$

Hence, If we use (2.11), (2.12) and (2.13) in (2.10), we obtain the desired result. This completes the proof. 
Remark 2.5. In Theorem 2.2, if we take $\alpha=1, \mu=1-\theta$ and $x=(1-\lambda) a+\lambda b$ with $\lambda \in[0,1]$, then then we obtain the same of the inequality (1.4) in Theorem 1.6 .

Corollary 2.7. In Theorem 2.2, if we take $s=1$, then the inequality (2.9) reduces to the following inequality

$$
\begin{aligned}
& \left|S_{f}(x, \mu, \alpha, a, b)\right| \\
\leq & A_{4}^{\frac{1}{p}}(\alpha, \mu, p)\left\{\frac{(x-a)^{\alpha+1}}{b-a}\left(\frac{\left|f^{\prime}(x)\right|^{q}+\left|f^{\prime}(a)\right|^{q}}{2}\right)^{\frac{1}{q}}\right. \\
+ & \left.\frac{(b-x)^{\alpha+1}}{b-a}\left(\frac{\left|f^{\prime}(x)\right|^{q}+\left|f^{\prime}(b)\right|^{q}}{2}\right)^{\frac{1}{q}}\right\} .
\end{aligned}
$$

Corollary 2.8. In Theorem 2.2, if we take $x=\frac{a+b}{2}$, then the inequality (2.9) reduces to the following inequality

$$
\begin{aligned}
& \frac{2^{\alpha-1}}{(b-a)^{\alpha-1}}\left|S_{f}\left(\frac{a+b}{2}, \mu, \alpha, a, b\right)\right| \\
= & \mid(1-\mu) f\left(\frac{a+b}{2}\right)+\mu\left(\frac{f(a)+f(b)}{2}\right)-\frac{\Gamma(\alpha+1) 2^{\alpha-1}}{(b-a)^{\alpha}}\left[J_{\left.\left(\frac{a+b}{2}\right)^{-} f(a)+J_{\left(\frac{a+b}{2}\right)^{+} f(b)}^{\alpha}\right] \mid}^{\leq}\right. \\
\leq & \frac{b-a}{4} A_{4}^{\frac{1}{p}}(\alpha, \mu, p)\left\{\left(\frac{\left|f^{\prime}\left(\frac{a+b}{2}\right)\right|^{q}+\left|f^{\prime}(a)\right|^{q}}{2}\right)^{\frac{1}{q}}\right. \\
& \left.+\left(\frac{\left|f^{\prime}\left(\frac{a+b}{2}\right)\right|^{q}+\left|f^{\prime}(b)\right|^{q}}{2}\right)^{\frac{1}{q}}\right\} .
\end{aligned}
$$

Corollary 2.9. In Theorem 2.2, if we take $x=\frac{a+b}{2}$ and $\mu=\frac{1}{3}$, then the inequality (2.9) reduces the following Simpson type inequality for fractional integrals

$$
\begin{aligned}
& \left|\frac{2^{\alpha-1}}{(b-a)^{\alpha-1}} S_{f}\left(\frac{a+b}{2}, \frac{1}{3}, \alpha, a, b\right)\right| \\
& =\left|\frac{1}{6}\left[f(a)+4 f\left(\frac{a+b}{2}\right)+f(b)\right]-\frac{\Gamma(\alpha+1) 2^{\alpha-1}}{(b-a)^{\alpha}}\left[J_{\left(\frac{a+b}{2}\right)^{-}}^{\alpha} f(a)+J_{\left(\frac{a+b}{2}\right)^{+}}^{\alpha} f(b)\right]\right| \\
& \leq \frac{b-a}{4} A_{4}^{\frac{1}{p}}\left(\alpha, \frac{1}{3}, p\right)\left\{\left(\frac{\left|f^{\prime}\left(\frac{a+b}{2}\right)\right|^{q}+\left|f^{\prime}(a)\right|^{q}}{s+1}\right)^{\frac{1}{q}}\right. \\
& \left.+\left(\frac{\left|f^{\prime}\left(\frac{a+b}{2}\right)\right|^{q}+\left|f^{\prime}(b)\right|^{q}}{s+1}\right)^{\frac{1}{q}}\right\}
\end{aligned}
$$


Corollary 2.10. In Theorem 2.2, if we take $x=\frac{a+b}{2}$ and $\mu=0$ then the inequality (2.9) reduces the following midpoint inequality for fractional integrals

$$
\begin{aligned}
& \left|\frac{2^{\alpha-1}}{(b-a)^{\alpha-1}} S_{f}\left(\frac{a+b}{2}, 0, \alpha, a, b\right)\right| \\
= & \mid f\left(\frac{a+b}{2}\right)-\frac{\Gamma(\alpha+1) 2^{\alpha-1}}{(b-a)^{\alpha}}\left[J_{\left(\frac{a+b}{2}\right)^{-}}^{\alpha} f(a)+J_{\left.\left(\frac{a+b}{2}\right)^{+} f(b)\right]}^{\alpha}\right)^{\frac{1}{q}}\left\{\left(\frac{\left|f^{\prime}\left(\frac{a+b}{2}\right)\right|^{q}+\left|f^{\prime}(a)\right|^{q}}{s+1}\right)^{\frac{1}{q}}\right. \\
\leq & \left.\left.\frac{b-a}{4}\left(\frac{1}{\alpha p+1}\right)^{\frac{1}{p}}\right)^{\frac{1}{q}}\right\} .
\end{aligned}
$$

Remark 2.6. In Corollary 2.10, if we take $\alpha=1$ and use the inequality (1.2), then we obtain the same of the inequality in [3, Theorem 2.3].

Corollary 2.11. In Theorem 2.2, if we take $\mu=1$ then the inequality (2.9) reduces the following trapezoid inequality for fractional integrals

$$
\begin{aligned}
& \left|S_{f}\left(\frac{a+b}{2}, 1, \alpha, a, b\right)\right| \\
= & \left|\frac{(x-a)^{\alpha} f(a)+(b-x)^{\alpha} f(b)}{b-a}-\frac{\Gamma(\alpha+1)}{b-a}\left[J_{x^{-}}^{\alpha} f(a)+J_{x^{+}}^{\alpha} f(b)\right]\right| \\
\leq & \left(\frac{\beta(p+1,1 / \alpha)}{\alpha}\right)^{\frac{1}{p}}\left\{\frac{(x-a)^{\alpha+1}}{b-a}\left(\frac{\left|f^{\prime}(x)\right|^{q}+\left|f^{\prime}(a)\right|^{q}}{s+1}\right)^{\frac{1}{q}}\right. \\
& \left.+\frac{(b-x)^{\alpha+1}}{b-a}\left(\frac{\left|f^{\prime}(x)\right|^{q}+\left|f^{\prime}(b)\right|^{q}}{s+1}\right)^{\frac{1}{q}}\right\} .
\end{aligned}
$$

Remark 2.7. In Corollary 2.11, if we choose $\alpha=1$, we obtain the same of the inequality in $[4$, Theorem 6$]$.

Corollary 2.12. Let the assumptions of Theorem 2.2 hold. If $\left|f^{\prime}(x)\right| \leq M$ for all $x \in[a, b]$ and $\mu=0$, then from the inequality (2.9) we get the following Ostrowski type inequality for fractional integrals

$$
\begin{aligned}
& \left|\left[\frac{(x-a)^{\alpha}+(b-x)^{\alpha}}{b-a}\right] f(x)-\frac{\Gamma(\alpha+1)}{b-a}\left[J_{x^{-}}^{\alpha} f(a)+J_{x^{+}}^{\alpha} f(b)\right]\right| \\
\leq & M\left(\frac{1}{\alpha p+1}\right)^{\frac{1}{p}}\left[\frac{2}{s+1}\right]^{\frac{1}{q}}\left[\frac{(x-a)^{\alpha+1}+(b-x)^{\alpha+1}}{b-a}\right]
\end{aligned}
$$

for each $x \in[a, b]$.

Remark 2.8. In Corollary 2.12, if we choose $\alpha=1$, we get the same inequality in $[2$, Theorem 3].

\section{REFERENCES}

[1] M. Abramowitz and I.A. Stegun (Eds.), Handbook of Mathematical Functions with Formulas, Graphs, and Mathematical Tables. Dover, New York, 1965. 
[2] M. Alomari, M. Darus and S.S. Dragomir, P. Cerone, Ostrowski type inequalities for functions whose derivatives are s-convex in the second sense. Appl. Math. Lett. 23 (2010), 1071-1076.

[3] M. Alomari, M. Darus and U.S. Kirmaci, Some inequalities of Hermite-Hadamard type for s-convex functions. Acta Math. Sci. 31B(4) (2011), 1643-1652.

[4] M. Avci, H. Kavurmaci and M.E. Ozdemir, New inequalities of Hermite-Hadamard type via $s$-convex functions in the second sense with applications. Appl. Math. Comput. 217 (2011), $5171-5176$.

[5] Z. Dahmani, On Minkowski and Hermite-Hadamard integral inequalities via fractional via fractional integration. Ann. Funct. Anal. 1(1) (2010), 51-58.

[6] S.S. Dragomir and S. Fitzpatrick, The Hadamard's inequality for $s$-convex functions in the second sense. Demonstratio Math. 32(4) (1999), 687-696.

[7] R. Gorenflo and F. Mainardi, Fractional calculus; integral and differential equations of fractional order. Springer Verlag, Wien, 1997, 223-276.

[8] H. Hudzik and L. Maligranda, Some remarks on s-convex functions. Aequationes Math. 48 (1994), 100-111.

[9] İ. İşcan, New estimates on generalization of some integral inequalities for $s$-convex functions and their applications. Int. J. Pure Appl. Math. 86 (4) (2013), 727-746.

[10] İ. İscan, Generalization of different type integral inequalitiesfor $s$-convex functions via fractional integrals. Appl. Anal. (2013), 1-17. doi:10.1080/00036811.2013.851785.

[11] S. Miller and B. Ross, An introduction to the Fractional Calculus and Fractional Differential Equations. John Wiley \& Sons, USA, 1993.

[12] M.E. Ozdemir, M. Avci and H. Kavurmaci, Hermite-Hadamard type inequalities for $s$-convex and $s$-concave functions via fractional integrals, arXiv preprint arXiv:1202.0380. (2012).

[13] J. Park, Generalization of some Simpson-like type inequalities via differentiable $s$-convex mappings in the second sense, Int. J. Math. Math. Sci. 2011 (2011), 13 pages, Article ID 493531. doi:10.1155/493531.

[14] I. Podlubni, Fractional Differential Equations. Academic Press, San Diego, 1999.

[15] J. Pečarić, F. Proschan and Y.L. Tong, Convex Functions, Partial Orderings and Statistical Applications. Academic Press Inc.,1992.

[16] M.Z. Sarıkaya and N. Aktan, On the generalization of some integral inequalities and their applications. Math. Comput. Modelling. 54 (2011), 2175- 2182.

[17] E. Set, New inequalities of Ostrowski type for mapping whose derivatives are $s$-convex in the second sense via fractional integrals. Computers and Math. with Appl. 63 (2012), 1147-1154.

[18] M.Z. Sarıkaya and H. Ogunmez, On new inequalities via Riemann-Liouville fractional integration. Abstr. Appl. Anal. 2012 (2012), 10 pages, Article ID 428983. doi:10.1155/2012/428983

[19] E. Set, M.E. Ozdemir and M.Z. Sarkaya, On new inequalities of Simpson's type for quasiconvex functions with applications. Tamkang J. Math. 43(3) (2012), 357-364.

[20] M.Z. Sarıkaya, E. Set and M.E. Ozdemir, On new inequalities of Simpson's type for $s$-convex functions. Computers and Math. with Appl. 60 (2010), 2191-2199.

[21] M.Z. Sarıkaya, E. Set, H. Yaldız and N. Başak, Hermite-Hadamard's inequalities for fractional integrals and related fractional inequalities. Math. Comput. Modelling. 57(9) (2013), 24032407. doi:10.1016/j.mcm.2011.12.048.

Department of Mathematics, Faculty of Arts and Sciences, Giresun University, GireSUN, TURKEY

E-mail address: imdat.iscan@giresun.edu.tr 